\title{
CORRIGENDUM
}

\section{Influence of caecectomy and source of dietary fibre or starch on excretion of endogenous amino acids by laying hens}

\author{
BY C. M. PARSONS \\ Vol. 51 (1984), no. 3
}

Page 541, Abstract, no. 4, lines 2-3

For the intestinal microflora had greater influence on amino acids excreted by caecectomized hens than on those excreted by intact hens.

Read the intestinal microflora had greater influence on amino acids excreted by intact hens than on those excreted by caecectomized hens. 